\title{
Enhanced Integral Equation Analysis of Multilayered Periodic Structures Useful for the Design of Reflectarray Antennas
}

\author{
R. Florencio \\ R. R. Boix \\ J. A. Encinar
}

\begin{abstract}
When reflectarray antennas are designed under the local periodicity assumption, the problem of the scattering of plane waves by multilayered periodic structures has to be solved many times. The Method of Moments (MoM) in the spectral domain is the numerical technique usually employed for the analysis of these multilayered structures. Unfortunately, it is not computationally efficient since it requires the determination of slowly convergent double infinite summations. In this paper the Mixed Potential Integral Equation (MPIE) formulation of the MoM in the spatial domain is invoked to transform the slowly convergent summations into singular finite double integrals that can be efficiently computed. The novel MoM approach in the spatial domain has been found to be between one and two orders of magnitude faster than the traditional spectral domain MoM both in the analysis of multilayered periodic structures, and in the design of reflectarray antennas with cell characterization based on the local periodicity assumption.
\end{abstract}

\section{INTRODUCTION}

Reflectarray antennas provide interesting advantages over reflector antennas and phased arrays, but narrow bandwidth is one their main limitations [1]. Multiresonant elements in multilayered configuration such as stacked rectangular patches have been introduced to increase the bandwidth of these antennas [1]. Also, stacked rectangular patches make it possible to design dual polarization reflectarray antennas with independent beamforming in each polarization [2]. Very recently, multilayered reflectarray elements containing two orthogonal sets of parallel dipoles have been proposed that keep the performance of the element based on stacked patches in terms of bandwidth and dual polarization, while requiring a simpler manufacturing process, and providing a lower level of crosspolarization [3].
In the design of multilayered reflectarray antennas, the sizes of the elements -patches or dipoleshave to be adjusted to obtain the appropriate reflection phases that lead to the generation of the desired radiation pattern. When choosing the sizes of the elements, it is customary to assume that each element is surrounded by an infinite periodic array of identical elements, which is known as the local periodicity assumption [1]. The design of the antennas under this assumption requires the electromagnetic analysis of a huge number of periodic arrays of patches/dipoles in a multilayered substrate $[1,2]$. The numerical method traditionally employed for the analysis of the periodic multilayered structures is the Method of Moments (MoM) in the spectral domain [4]. Unfortunately, this method may not be fast enough for reflectarray design purposes since the determination of the MoM matrix entries requires the computation of slowly convergent double infinite summations when test and basis functions belong to metallizations placed at the same interface of the multilayered substrate. In this paper we present a faster and more accurate hybrid spectral/spatial method in which the specific slowly convergent summations are computed by means of a spatial domain formulation of the MoM. In particular, the MoM matrix entries are expressed as double integrals containing multilayered periodic Green's functions (MPGF) times cross-correlations of the basis functions. The spatial domain MPGF for the potentials are interpolated with great accuracy in terms of 2-D Chebyshev polynomials after extracting in closed-form the singular behavior around the source points [5]. Also, the logarithmic singularities introduced by the cross-correlations in the integrals are numerically handled by means of Ma-Rokhlin-Wandzura (MRW) quadrature rules [6]. The new hybrid spectral/spatial MoM has been found to be between one and two orders of magnitude faster than the traditional spectral domain MoM in the analysis of multilayered periodic structures containing either patches or dipoles for an accuracy of $1 \%$. Similar CPU time savings have been obtained when the new hybrid MoM code has been used for the design of a dual-polarization reflectarray antenna made of stacked patches [2]. 


\section{Outline of the numerical procedure}

Fig. 1 shows the side and top views of a multilayered periodic structure in which the unit cell contains three stacked rectangular patches. The multilayered substrate consists of $N_{l}$ lossy dielectric layers of thickness $d_{i}$ and complex permittivity $\varepsilon_{i}=\varepsilon_{0} \varepsilon_{r, i}\left(1-j \tan \delta_{i}\right)\left(i=1, \ldots, N_{l}\right)$. The unit cell of the multilayered periodic structure is that of the reflectarray element used for the design of the dual-polarization reflectarray antenna of [2]. Alternative unit cells containing parallel coplanar dipoles at different metallization levels are required for the design of the reflectarray antennas of [3]. Let us assume the periodic structure is illuminated by a plane wave with incidence direction given by the angular spherical coordinates $\theta_{\mathrm{i}}$ and $\varphi_{\mathrm{i}}[5]$. In order to design a reflectarray antenna, we need to determine the scattering matrix relating the cartesian $x$ and $y$ components of the incident electric field and the reflected electric field (in the specular direction) at the plane $z=0$ (see Eqn. (3.25) of [1]). The computation of the reflected field requires to know the current density on the patches. If the patches are assumed to be perfect electric conductors (PEC), the current density on the patches is the solution of the following set of three coupled electric field integral equations (EFIE's)

$$
\begin{aligned}
& \hat{\mathbf{z}} \times\left[\mathbf{E}^{\mathrm{ms}}\left(x, y, z=-h_{P_{i}}\right)\right. \\
&+ \sum_{j=1}^{3} \sum_{m=-\infty}^{+\infty} \sum_{n=-\infty}^{+\infty} \int_{a / 2-a_{j} / 2+m a}^{a / 2+a_{j} / 2+m a} \int_{b / 2-b_{j} / 2+n b}^{b / 2+b_{j} / 2+n b} \\
& \overline{\mathbf{G}}^{E}\left(x-x^{\prime}, y-y^{\prime}, z=-h_{P_{i}}, z^{\prime}=-h_{P_{j}}\right) \\
&\left.\cdot \mathbf{J}_{j}\left(x^{\prime}, y^{\prime}\right) d x^{\prime} d y^{\prime}\right]=\mathbf{0} \quad a / 2-a_{i} / 2<x<a / 2 \\
&+a_{i} / 2 ; b / 2-b_{i} / 2<y<b / 2+b_{i} / 2 \quad(i=1,2,3)
\end{aligned}
$$

where $\mathbf{J}_{j}(x, y)$ is a Floquet-periodic function that stands for the current density induced on the patches located at $z=-h_{P_{j}}(j=1,2,3)$, $\mathbf{E}^{\mathrm{ms}}(x, y, z)$ is the electric field generated in all space by the plane wave impinging on the multilayered substrate in the absence of the patches, and $\overline{\mathbf{G}}^{E}$ is the non-periodic dyadic Green's function of the multilayered substrate. In order to solve the EFIE's of (1), we approximate $\mathbf{J}_{j}(x, y)$ in terms of Floquet-periodic basis functions as

$$
\mathbf{J}_{j}(x, y)=\sum_{l=1}^{N_{b}} c_{j l} \mathbf{J}_{j l}(x, y) \quad(j=1,2,3)
$$

where the coefficients $c_{j l}\left(j=1,2,3 ; l=1, \ldots, N_{b}\right)$ are unknown coefficients. When (2) is substituted

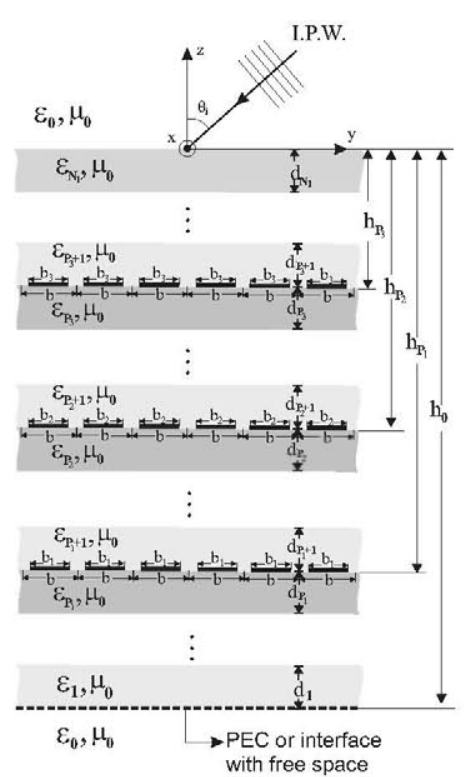

(a)

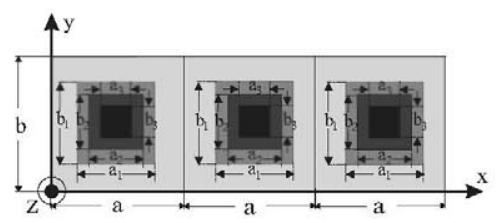

(b)

Figure 1: Side view (a) and top view (b) of a periodic multilayered structure. The unit cell contains three stacked rectangular patches. The periodic structure is illuminated by a plane wave.

into (1) and the Galerkin's version of the MoM is applied, a system of linear equations for the unknown coefficients $c_{j l}$ is obtained. By applying Parseval's identity for Fourier transforms to the entries of the MoM matrix, these entries can be expressed in the spectral domain as

$$
\begin{aligned}
& \Lambda_{k l}^{i j}=a b \sum_{m=-\infty}^{+\infty} \sum_{m=-\infty}^{+\infty}\left[\left(\widetilde{\mathbf{J}}_{i k}^{\mathrm{d}}\left(k_{x m}, k_{y n}\right)\right)^{*}\right]^{t} \\
& \tilde{\overline{\mathbf{G}}}^{E, \mathrm{c}}\left(k_{x}=k_{x m}, k_{y}=k_{y m}, z=-h_{P_{i}}, z^{\prime}=-h_{P_{j}}\right) \\
& \widetilde{\mathbf{J}}_{j l}^{\mathrm{d}}\left(k_{x m}, k_{y n}\right) \quad\left(i, j=1,2,3 ; k, l=1, \ldots, N_{b}\right)
\end{aligned}
$$

where $k_{x m}=\frac{2 \pi m}{a}+k_{0} \sin \theta_{\mathrm{i}} \cos \varphi_{\mathrm{i}}$ and $k_{y n}=\frac{2 \pi n}{b}+$ $k_{0} \sin \theta_{\mathrm{i}} \sin \varphi_{\mathrm{i}}\left(k_{0}=\omega \sqrt{\mu_{0} \varepsilon_{0}}\right)$, where $\widetilde{\mathbf{J}}_{j l}^{\mathrm{d}}\left(k_{x m}, k_{y n}\right)$ stand for the two-dimensional discrete Fourier's transforms of $\mathbf{J}_{j l}(x, y)$, and where $\widetilde{\overline{\mathbf{G}}}^{E, c}\left(k_{x}=\right.$ $\left.k_{x m}, k_{y}=k_{y m}, z=-h_{P_{i}}, z^{\prime}=-h_{P_{j}}\right)$ are samples of the two-dimensional Fourier transform of $\overline{\mathbf{G}}^{E}\left(x, y, z=-h_{P_{i}}, z^{\prime}=-h_{P_{j}}\right)$. It can be analytically shown that the summations involved in the computation of $\Lambda_{k l}^{i j}$ show a convergence of the type 
$\mathrm{e}^{-\sqrt{k_{x m}^{2}+k_{y n}^{2}}\left|h_{P_{i}}-h_{P_{j}}\right|}$ as $k_{x m}^{2}+k_{y n}^{2} \rightarrow \infty$. In practical cases, a few hundred terms suffice to compute the series of (3) with a great degree of accuracy when $i \neq j$. However, several million terms may be required for the accurate computation of the series of (3) when $i=j$. To avoid this lengthy computation, in this paper the MoM entries $\Lambda_{k l}^{i i}(i=1,2,3)$ have been computed in the spatial domain as

$$
\begin{aligned}
\Lambda_{k l}^{i i}= & -j \omega T_{i k l}^{A}-\frac{1}{j \omega} T_{i k l}^{\phi} \\
& \quad\left(i=1,2,3 ; k, l=1, \ldots, N_{b}\right)
\end{aligned}
$$

The quantities $T_{i k l}^{A}$ and $T_{i k l}^{\phi}$ of (4) are given by the 2-D integrals

$$
\begin{aligned}
& T_{i k l}^{A}=\int_{-a_{i}}^{a_{i}} \int_{-b_{i}}^{b_{i}} g_{i k l}^{A}(x, y) \\
& \times G_{x x}^{A, p p}\left(x, y, z=-h_{P_{i}}, z^{\prime}=-h_{P_{i}}\right) d x d y \\
& T_{i k l}^{\phi}=\int_{-a_{i}}^{a_{i}} \int_{-b_{i}}^{b_{i}} g_{i k l}^{\phi}(x, y) \\
& \times G^{\phi, \operatorname{pp}}\left(x, y, z=-h_{P_{i}}, z^{\prime}=-h_{P_{i}}\right) d x d y
\end{aligned}
$$

where the functions $G_{x x}^{A, p p}$ and $G^{\phi, p p}$ are MPGF with 2-D periodicity for the vector potential and scalar potential of the multilayered substrate of Fig. 1, where $g_{i k l}^{A}(x, y)$ is a 2-D cross-correlation between $\mathbf{J}_{i k}(x, y)$ and $\mathbf{J}_{i l}(x, y)$, and where $g_{i k l}^{\phi}(x, y)$ is a 2-D cross-correlation between $\nabla \cdot \mathbf{J}_{i k}(x, y)$ and $\nabla \cdot \mathbf{J}_{i l}(x, y)[5]$. The MPGF $G_{x x}^{A, \mathrm{pp}}$ and $G^{\phi, \mathrm{pp}}$ can be expressed in terms of slowly convergent double infinite summations. In this paper, important CPU time reductions have been achieved in the computation of $G_{x x}^{A, p p}$ and $G^{\phi, p p}$ by interpolating these two functions. In principle, this interpolation is seriously affected by the singularities of $G_{x x}^{A, \mathrm{pp}}$ and $G^{\phi, \mathrm{pp}}$ caused by the source point, and by the quasi-singularities caused by the quasidynamic images of the source trough the nearest layers. To avoid this deleterious effect on the interpolation, both the source singularities and the images quasi-singularities have been analytically extracted prior to the interpolation. The terms containing the source singularities and the images quasi-singularities of $G_{x x}^{A, p p}$ and $G^{\phi, p p}$ have been estimated as the the inverse 2-D Fourier transform of the complex image exponential approximation of the asymptotic spectral MPFG [7]. Let $G_{0 i}^{\mathrm{pp}}(x, y)(i=1,2,3)$ represent any of the two MPGF $G_{x x}^{A, p p}\left(x, y, z=-h_{P_{i}}, z^{\prime}=-h_{P_{i}}\right)$ and $G^{\phi, \operatorname{Pp}}\left(x, y, z=-h_{P_{i}}, z^{\prime}=-h_{P_{i}}\right)$ of $(5)$ and $(6)$, and let $G_{0 i}^{\mathrm{pp} \text {,sing }}(x, y)$ represent the term containing the singularities and quasi-singularities of $G_{0 i}^{\mathrm{pp}}(x, y)$. In this paper, $G_{0 i}^{\mathrm{pp}}(x, y)-G_{0 i}^{\mathrm{pp}, \text { sing }}(x, y)$ have been interpolated in terms of 2-D Chebyshev polynomials as shown below

$$
\begin{aligned}
& G_{0 i}^{\mathrm{pp}}(x, y)-G_{0 i}^{\mathrm{pp}, \operatorname{sing}}(x, y) \\
& \approx \sum_{m=0}^{N_{c}} \sum_{n=0}^{N_{c}} h_{m n}^{i} T_{m}\left(\frac{2 x}{a}-1\right) T_{n}\left(\frac{2 y}{b}-1\right)
\end{aligned}
$$

Numerical simulations have shown that $N_{c} \approx 7$ suffices to provide $G_{0 i}^{\mathrm{pp}}(x, y)-G_{0 i}^{\mathrm{pp}, \text { sing }}(x, y)$ with an accuracy of four significant figures in $(7)$.

The basis functions $\mathbf{J}_{j l}(x, y)(j=1,2,3)$ used in this paper are products of Chebyshev polynomials weighted by functions which account for edge singularities (see Eqn. (A.13) of [8]) since these basis functions ensure a quick convergence of the MoM with respect to the number of basis functions. For these particular basis functions, the 2-D integrals of (5) and (6) can be expressed as linear combinations of integrals of the type

$$
A_{p q, r s}^{i}=\int_{-a_{i}}^{a_{i}} \int_{-b_{i}}^{b_{i}} t_{p r}(x) t_{q s}(y) G_{0 i}^{\mathrm{pp}}(x, y) d x d y
$$

where $t_{p r}(x)$ and $t_{q s}(y)$ can be expressed as linear combinations of complete elliptic integrals of first and second kind (see Eqn. (45) of [5]). The integrands of (8) contain both Green's function $1 / \rho=$ $1 / \sqrt{x^{2}+y^{2}}$ singularities and logarithmic singularities introduced by the elliptic functions (see Fig. 3), which has a deleterious effect on the numerical computation of the integrals $T_{i k l}^{A}$ and $T_{i k l}^{\phi}$. In this paper, the $1 / \rho$ singularities have been extracted and integrated in quasi-closed form. Also, the logarithmic singularities have been accurately handled by means of MRW quadrature rules [6]. In the case where coplanar dipoles are part of the periodic unit cell (this is required for the design of the reflectarray antennas of [3]), the integrals of (5) and (6) for test and basis functions on different dipoles still keep the logarithmic singularities (that can be handled by MRW quadrature rules) but do not include the $1 / \rho$ singularity of Fig. 3 .

\section{Numerical results}

In Figs. 3(a) and (b) we plot results for the power reflection and transmission coefficients of the first grating lobe $m=-1, n=0$ generated by a periodic array of three stacked dipoles in free space (see Fig. 2 of [9]). In order to validate our MoM codes for the analysis of the scattering by the structure of Fig. 1(a), in Figs. 3(a) and (b) our results obtained with both the standard spectral MoM and the hybrid spectral-spatial MoM are compared with those 


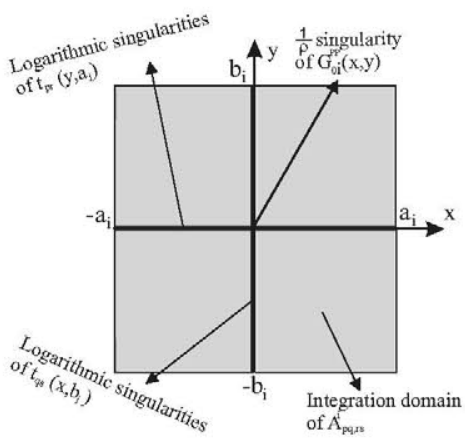

Figure 2: Integration domain of the integrals of (8) (grey rectangle), and singularities of the integrand.

of Fig. 6 of [9]. Good agreement is observed between our two sets of MoM results, and reasonable agreement is obtained between our results and the results of [9]. The CPU time required by the hybrid MoM for the generation of the curves of Figs. 3(a) and (b) was around 60 times smaller than that required by the spectral MoM for an accuracy of two significant figures. As a further check on the efficiency of the new hybrid spectral-spatial MoM approach, the contoured beam reflectarray antenna made of stacked patches of [2] has been designed with both the standard spectral MoM code for periodic structures and the novel hybrid spectralspatial MoM code at two frequencies, $11.575 \mathrm{GHz}$ for $\mathrm{V}$-polarization and $12.1 \mathrm{GHz}$ for $\mathrm{H}$-polarization. It has been found that the CPU time required in the design of the reflectarray with the hybrid MoM code is around 30 times smaller than that required by the spectral MoM code, which demonstrates the applicability of the novel MoM approach presented in this paper in the design of reflectarray antennas.

\section{Acknowledgments}

This work has been supported by Junta de Andalucía (project TIC-1435), by European Space Agency under ESTEC Contract No. 4000106334, and by the Spanish Ministry of Science and Innovation (projects CICYT TEC2013-43345-P and CICYT TEC2013-41913-P).

\section{References}

[1] J. Huang and J. A. Encinar, Reflectarray antennas. Piscataway, NJ/New York: IEEE Press/Wiley, 2008.

[2] J. A. Encinar, L. Datashvili, J. A. Zornoza, M. Arrebola, M. Sierra-Castaer, J. L. Besada, H. Baier, and H. Legay, "Dual-polarization dual-coverage reflectarray for space spplications," IEEE Trans.

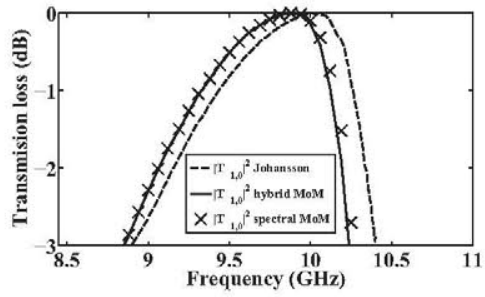

(a)

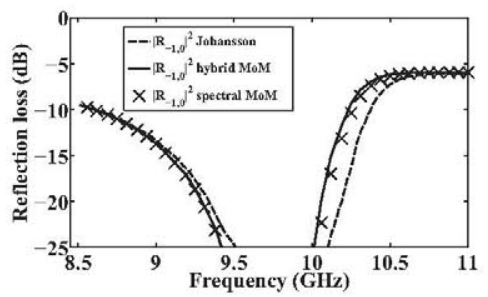

(b)

Figure 3: Transmission loss (a) and reflection loss (b) of the diffracted $(-1,0)$ mode for the free space frequency scanned reflection grating of Fig. 6 of [9].

on Antennas and Propag., Vol. 54, pp. 2828-2837, Oct. 2006.

[3] R. Florencio, J. A. Encinar, R. R. Boix, V. Losada, G. Tosso, IEEE Trans. on Antennas and Propag., Vol. 63, pp. 1234-1246, Apr. 2015.

[4] R. Mittra, C. H. Chan, and T. Cwik, "Techniques for analyzing frequency selective surfaces-A review", Proc. IEEE. vol. 76, pp. 1593-1615, Dec. 1988.

[5] R. Florencio, R. R. Boix, and J. A. Encinar, "Enhanced MoM analysis of the scattering by periodic strip gratings in multilayered substrates," IEEE Trans. on Antennas and Propag., Vol. 61, pp. 5088-5099, Oct. 2013.

[6] F. Vipiana, D. R. Wilton, and W. A. Johnson, "Advanced numerical schemes for the accurate evaluation of 4 -D reaction integrals in the method of moments", IEEE Trans. on Antennas and Propag., Vol. 61, pp. 5559-5566, Nov. 2013.

[7] M. I. Aksun, "A robust approach for the derivation of closed-form Green's functions," IEEE Trans. Microwave Theory Tech., vol. 44, pp. 651-658, May 1996.

[8] A. M. Lerer and A. G. Schuschinsky, "Full-wave analysis of three-dimensional planar structures," IEEE Trans. Microwave Theory Tech., vol. 41, pp. 2002-2015, Nov. 1993.

[9] F. S. Johansson, "Frequency-scanned gratings consisting of photo-etched arrays," IEEE Trans. Antennas Propagat., vol. 37, pp. 996-1002, Aug. 1989. 